\title{
SUBLINEAR FUNCTIONALS AND KNOPP'S CORE THEOREM
}

\author{
C. ORHAN \\ Department of Mathematics, Faculty of Scicnc.. \\ University of Ankara, Ankara-()(Gl()), TLRKF:Y \\ (Received January 26, 1989 and in revised form October 20, 1989)
}

ABSTR $\Lambda C T$. In this paper we are concerned with inequalities involving certain sublinear functionals on $m$, the space of real bounded sequences. Such inequalitics being analogues of Knopp's (orre theorem.

KI:Y W(ORI)S ANI) PIIRASES. Core theorem, Sublincar functionals, Infinite matrices, Almosi convergence.

198() ^MS CL,ASSIFFICATION CODES. 40C(05, 40J(05, 46^45

\section{INTRODUCTION.}

Let $\mathrm{m}$ he the linear space of real bounded sequences with the usual supremum nom. We write

$$
m_{0}=\left\{x \in m: \sup _{n}\left|\sum_{k=0}^{n} x_{k}\right|<\infty\right\}
$$

Let $\lambda$ be the sequence of infinite matrices $\left(A^{i}\right)=\left(a_{n k}(i)\right)$. Given a sequence $x=\left(x_{k}\right)$ we write

$$
A_{n}^{i}(x)=\sum_{k=0}^{\infty} a_{n k}(i) x_{k}
$$
if it exists for each $n$ and $i \geq 0$. We also write $A x$ for $\left(A_{n}^{i}(x)\right)_{i, n=0}^{\infty}$. The sequence $x=\left(x_{k}\right)$ is said
to be summable to the value $s$ by the method $(\lambda)$ if

$$
A_{n}^{i}(x) \rightarrow s \quad(n \rightarrow \infty, \text { uniformly in } i)
$$

If (1.2) holds, then we write $x \rightarrow s(\mathcal{A})$.

If we define $\left(a_{n k}(i)\right.$ by

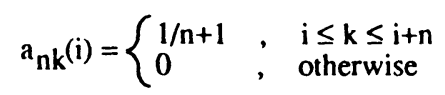

then $(\mathcal{A})$ reduces to the method $f($ Lorentz [1]). In the case

$$
a_{n k}(i)=\frac{1}{n+1} \sum_{r=i}^{i+n} a_{r k}
$$

( $\lambda$ ) reduces to the almost summability method (King |2|). If $\lambda=\lambda=\left(a_{n k}\right)$, then we ge(t) the usual summability method (A). 


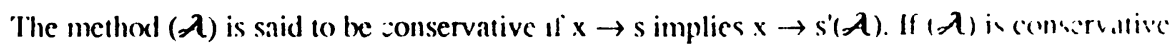
and $s=s^{\prime}$, then $(\boldsymbol{\lambda})$ is called regular.

It is well known, (Sticglitz |3|), that $(\lambda)$ is regular if and only if the following conditions hold:

$$
\sum_{k}\left|a_{n k}(i)\right|<\infty \quad, \quad(\text { for all } n, \text { for all } i)
$$

and there exist an integer $m$ such that

$$
\begin{gathered}
\sup _{i \geq(), n \geq m} \sum_{k}\left|a_{n k}(i)\right|<\infty \\
\lim _{n} a_{n k}(i)=0, \quad \text { unifornly in i, } \\
\lim _{n} \sum_{k} a_{n k}(i)=1, \quad \text { uniformly in } i .
\end{gathered}
$$

Throughout the palper we write

$$
\|\not \lambda\|=\sup _{n, i} \sum_{k}\left|a_{n k}(i)\right|<\infty
$$

to mean that, there exists a constant $M$ such that

$$
\left.\sum_{k}\left|a_{n k}(i)\right| \leq M \quad \text { (for all } n, \text { for all } i\right)
$$

and the scries

$$
\sum_{k} a_{n k}(i)
$$

convreges uniformly in $\mathrm{i}$ for each $\mathrm{n}$.

If, for every bounded secjuence $x, x \rightarrow s(\mathcal{A})$ then $(\mathcal{A})$ is said to be a Schur method

Throughout the paper we consider only real matrices and real bounded sequences.

In this paper we are concerned with inequalities involving certain sublinear functionals on $\mathrm{m}$. the space of real bounded sequences. Such inequalities being analogues of Knopp's ('ore thenrem That theorem detcrnines a class of regular matrices for which

\section{limsup Ax $\leq$ limsup $x$}

for all $x \in m$, see e.g Cooke [4], Maddox [5], Simons [6]. This result has also been extended to coregular matrices by Rhoades [7], Schaefer [8], and, Das [9].

Before stating the theorems to be proved, we introduce some further notation.

$$
\begin{aligned}
& C(x)=\liminf _{n} ; L(x)=\operatorname{limsupx}_{n},\|x\|=\sup \left|x_{n}\right| \\
& \mathcal{L}^{*}(x)=\liminf _{n} \sup _{i} \frac{1}{n+1} \sum_{r=i}^{i+n} x_{r} \\
& L^{*}(x)=\limsup _{n} \sup _{i} \frac{1}{n+1} \sum_{r=i}^{i+n} x_{r} \\
& =\inf _{z \in m_{0}} L^{*}(x+z)
\end{aligned}
$$


If $f$, g are any two of the above functionals, we shall write $f A \leq g B$ to denote that, for every beunded sepucince $x$, the transforms $A x$ and $B x$ are defined and boundeil and $f(A x) \leq g(B x)$.

2. IIII MIIIN RI:SUITS.

We wrike, lor $x \in$ : $m$,

$$
Q_{\lambda}(x)=\limsup _{n} \sup _{i} \sum_{k} a_{n k}(i) x_{k}
$$

and

$$
q_{A}(x)=\liminf _{n} \sup _{i} \sum_{k} a_{n k}(i) x_{k}
$$

Wih thas notation we have

TIII:(ORI:M.1. Let $\|\mathcal{A}\|<\infty$. Then

$$
\mathrm{Q}_{\mathrm{A}} \leq \mathrm{L}
$$

11 and only if $(\mathcal{A})$ is regular and

$$
\sum_{k}\left|a_{n h}(i)\right| \rightarrow 1 \quad(n \rightarrow \infty, \text { uniformlly in i) }
$$

PR( () $\mid$ : Necessity. I et $x=\left(x_{k}\right)$ be a convergent sefuence. Then $[(x)=I,(x)=\lim x$. By (2.1), we have

$$
\mathcal{L}(x) \leq-Q_{A}(-x) \leq Q_{A}(x) \leq L(x) .
$$

Hence we get that $Q_{A}(x)=q_{A}(x)=\lim x$. So $(\mathcal{A})$ is regular.

Since (A) is regular, the reguirement of Lemma 2, (Das $[9 \mid$ ), is satisfied. Hence there cisists $y \in m$ such that $\|y\| \leq 1$ and

$$
Q_{A}(y)=\underset{n}{\limsup } \sup _{i} \sum_{k}\left|a_{n k}(i)\right|
$$

Hence, lakmg $\lambda=c=(1,1, \ldots)$, we have

$$
\begin{aligned}
& 1=\varphi_{A}(c) \leq \liminf _{n} \sup _{i} \sum_{k}\left|a_{n h}(i)\right| \\
& \leq \underset{n}{\limsup } \sup _{i} \sum_{k}\left|a_{n k}(i)\right|=Q_{A}(y) \leq L(y) \leq\|y\| \leq 1
\end{aligned}
$$

which proves the necessity of (2.2)

Sulficiency. We define, for any real $\lambda, \lambda^{+}=\max \left(\lambda,(0), \lambda^{-}=\operatorname{man}(-\lambda,())\right.$. Then $|\lambda|-\lambda^{+}+\lambda^{-}$ and $\Lambda-\lambda^{1}-\lambda^{-}$. Hence

$$
\sum_{h} a_{n k}(i) x_{k}=\sum_{k<m} a_{n k}(i) x_{k}+\sum_{k \geq m}\left(a_{n k}^{+}(i)\right) x_{k}-\sum_{k \geq m}\left(a_{n k}^{-}(i)\right) x_{k}
$$

So we have

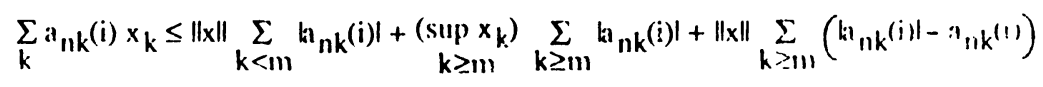

By hypothesis, we get that $Q_{\Lambda}(x) \leq L(x)$.

REMARK. We could use Theorem 2, (Das $|9|$ ), to get the sufficiency. 
C.OR()I.I.ARY.2. We have on $\mathrm{m}$,

$$
\mathfrak{l} \leq \mathfrak{L}^{*} \leq \mathrm{L} \boldsymbol{L}^{*} \leq \mathrm{l}
$$

PRO()I: In theorem 1, it is enough to take

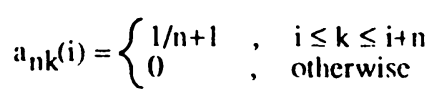

We deduce at once from Corollary 2 that if a sequence $x$ is convergent to s. then it is almoul convergent to $\mathrm{s}$ which is a well-known result.

We note that, by considering Theorem 1 , one may get necessary and sufficient comditions lon $I . * A \leq I$, and $I . A \leq I$.

In the next theorem we consider the inequality $\mathrm{L}, \mathrm{\Lambda} \leq \mathrm{I}$ **

THIEOREM.3. $\mathrm{LA} \leq \mathrm{I}$, $^{*}$ if and only if $\mathrm{A}$ is strongly regular and

$$
\sum_{k} \mathbf{a}_{n k} \mid \rightarrow 1 \quad(n \rightarrow \infty)
$$

PROOF. Recall that a matrix $A$ is called strongly regular if it maps all almost comvergent sceuences into the convergent sequences and $\lim \Lambda x=f-\lim x$.

We first prove the necessity. It is easy to see that $L^{*} \leq L \Lambda \leq I, \Lambda \leq L^{*}$. If $x$ is almost converge then $f-\lim x=\mathcal{L}(x)=L^{*}(x)$. Hence, by the hypothesis, $L(\Lambda x)=L(\Lambda x)=f-\lim x$. So $A$ is strongly regular. Using the fact that $L^{*} \leq \mathrm{L}$, (see Corollary 2 ) and that $L A \leq L^{*}$, we get that $L, A \leq 1$. Now the necessity of (2.3) follows from Knopp's Core theorem (sce, c.g. Maddox |5|). and that

We note in passing that a matrix $A$ is strongly regular, Lorentz $|1|$, if and only if it is regular

$$
\sum_{k} h_{n k}-a_{n, k+1} \rightarrow 0 \quad(n \rightarrow \infty)
$$

Sufficiency. Given $\varepsilon>0$, we can find a positive integer $p$ such that for $x \in m$ and for all $\mathrm{k} \geq \mathbf{0}$

$$
\frac{1}{p+1} \sum_{r=k}^{k+p} x_{r}<L^{*}(x)+\varepsilon
$$

(We fix p throughout the analysis).

As in Lorentz's proof (see [1]; Th. 7) one can show that

$$
\begin{aligned}
\sum_{k=0}^{\infty} a_{n k} x_{k} & =\sum_{k=0}^{\infty} a_{n k} \frac{1}{p+1} \sum_{r=k}^{k+p} x_{r} \\
& -\sum_{k=p}^{\infty}\left(\frac{a_{n k}+\ldots+a_{n, k-p}}{p+1}-a_{n k}\right) \\
& +\sum_{k=0}^{p-1} a_{n k} x_{k} \\
& +\sum_{k=0}^{p-1}\left(\frac{a_{n k}+\ldots+a_{n, k-p}+1}{p+1}\right) x_{k}
\end{aligned}
$$


Sinci $\lambda \in m$, it follows from the regularity of $A$ that the third and fourth sigmas in (2.6) tend (1) cal as $n \rightarrow \infty$. If we write

$$
I_{n p}=-\sum_{k=p}^{\infty}\left(\frac{a_{n k}+\ldots+a_{n, k}-\underline{p}}{p+1}-a_{n k}\right) x_{k}
$$

then

$$
\begin{aligned}
\left|F_{n p}\right| & \leq \frac{1}{p+1} \sum_{k=p}^{\infty} a_{n k}+\ldots+a_{n, k-p}-(p+1) a_{n k}\left|x_{k}\right| \\
& \leq \frac{\|x\|}{p+1} \sum_{r=0}^{p} \sum_{k=p}^{\infty} a_{n, k-r}-a_{n k} \mid \\
& \leq \frac{\|x\|}{p+1} \sum_{r=0}^{p} r \sum_{k=0}^{\infty} a_{n k}-a_{n, k+1} \mid \\
& \leq \frac{p}{2}\|x\| \sum_{k=0}^{\infty} a_{n k}-a_{n, k+1} \mid
\end{aligned}
$$

Since A is strongly regular, (2.4) holds. Thus the expression in (2.7) tends to zero as $n \rightarrow \infty$. Hence we find that

$$
\begin{aligned}
L .(A x) & \leq \limsup _{n} \sum_{k=0}^{\infty} a_{n k}\left(\frac{x_{k}+\ldots+x_{k+p}}{p+1}\right) \\
& \leq \limsup _{n} \sum_{h}\left(a_{n k}^{+}\right)\left(\frac{x_{k}+\ldots+x_{k+p}}{p+1}\right) \\
& -\underset{n}{\limsup } \sum_{k}\left(a_{n k}^{-}\right)\left(\frac{x_{k}+\ldots+x_{k+p}}{p+1}\right)
\end{aligned}
$$

By (2.5), we have

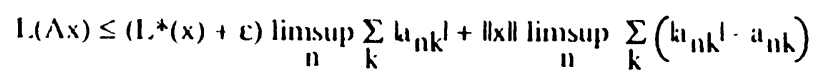

Using the regilarity of $\mathrm{A}$ and (2.3) we get that

$$
L(A x) \leq L^{*}(x)+\varepsilon .
$$

Since $\varepsilon$ is anbitrary', sufficiency follows.

TIII:OREM.4. $L^{*} A \leq 1$.* if and only if $A$ is F-regular and

$$
\lim _{n} \sup _{i} \sum_{k}\left|\frac{1}{n+1} \sum_{r=i}^{i+n} a_{r k}\right|=1
$$

PROOI: Recall that $A$ is called F-regular if it maps $F$, the class of all almost convergent sepuences, into itself and $f-\lim A x=f-\lim x$. Corollary to Theorem 4 in $|10|$ gives the necessary and sufficient conditions for $A$ to be F-regular.

We now come to the proof of necessity.

One can easily show that 


$$
l *(x) \leq l *(A x) \leq L^{*}(A x) \leq L^{*}(x)
$$

If $x \in I^{\prime}$, then $L^{*}(\lambda)=L^{*}(x)=f-\lim x$. Hence $L^{*}(A x)=L^{*}(A x)=f-\lim x$. So $A$ is 1. regular.

fi) get the necessity of (2.8), we define $\left(b_{n k}(i)\right)$ by

$$
b_{n k}(i)=\frac{1}{n+1} \sum_{r=i}^{i+n} a_{r k}
$$

Observe now that the conditions of Lemma 2, Das 191 , are satisfied. So we must have a bounded seyuence $y$ such that $\|y\| \leq 1$ and

$$
Q_{13}(y)=\underset{n}{\limsup } \sup _{i} \sum_{k} \|_{n k}(i) \mid
$$

Hence by (2.9) and F-regularity of $A$, we get

$$
\begin{aligned}
1 & \leq \underset{n}{\liminf } \sup _{i} \sum_{k}\left|\frac{1}{n+1} \sum_{r=i}^{i+n} a_{r k}\right| \\
& \leq \underset{n}{\limsup \sup _{i}} \sum_{k} \frac{1}{n+1} \sum_{r=i}^{i+n} a_{r k} \mid \\
& =\underset{n}{\limsup \sup _{i}} \sum_{h}\left(\frac{1}{n+1} \sum_{r=i}^{i+n} a_{r k}\right) y_{k} \leq 1 *(y) \leq\|y\| \leq 1
\end{aligned}
$$

"whi pures (2.8).

Sulticiency. We first note that

$$
\begin{aligned}
L^{*}(A x) & =\limsup _{n} \sup _{i} \frac{1}{n+1} \sum_{r=i}^{i+n} A_{r}(x) \\
& =\underset{n}{\limsup \sup } \sum_{k}\left(1-1 \sum_{r=i}^{i+n} a_{r k}\right) x_{k}
\end{aligned}
$$

If we sel

$$
b_{n k}(i)=\frac{1}{n+1} \sum_{r=i}^{i+n} a_{r k}
$$

then (2.6) with $a_{n k}$ relaced by $b_{n k}(i)$ holds. Since $x \in m$ and $A$ is F-regular, Corollary to Theorem 1 in $|10|$ yields that the second and third signas with $a_{n k}$ replaced by $b_{n k}(i)$, tend to zero as $n \rightarrow \infty$, uniformly in i. On the other hand $I_{n p}$, with $a_{n k}$ replaced by $b_{n k}(i)$ is not greater than

$$
\frac{p}{2}\|x\| \sum_{k} \mid b_{n k}(i)-b_{n, k+1}(i) l=\frac{p}{2}\|x\| \sum_{k} \frac{1}{n+1} \sum_{r=i}^{i+n}\left(a_{r k}-a_{r, k+1}\right) \|
$$

Since $A$ is $F$-regular, the last sigma tends to zero as $n \rightarrow \infty$, uniformly in i. Hence we have. by (2.5). that

$$
L^{*}(A x) \leq \limsup _{n} \sup _{i} \sum_{k}\left(\frac{1}{n+1} \sum_{r=i}^{i+n} a_{r k}\right)\left(\frac{x_{k}+\ldots+x_{k+p}}{p+1}\right)
$$




$$
\begin{aligned}
& \leq\left(L^{*}(x)+\varepsilon\right) \underset{n}{\limsup \operatorname{supp}_{i}} \sum_{k}\left|\frac{1}{n+1} \sum_{r=i}^{i+n} a_{r k}\right| \\
& +\|x\| \underset{n}{\limsup } \sup _{i} \sum_{k}\left(\frac{1}{n+1} \sum_{r=i}^{i+n} a_{r k} \mid-\frac{1}{n+1} \sum_{r=i}^{i+n} a_{r k}\right)
\end{aligned}
$$

Using (2.8) and the fact that $\Lambda$ is F-regular, we get

$$
L^{*}(A x) \leq L^{*}(x)+\varepsilon .
$$

Since $\varepsilon$ is arhitrary, the required conclusion follows.

We now give another ingepuality sharper than that of Theorem 4 . (Sec Theorem $6 / x \cdot(1,1)$ is also an analoguc of Theorem 3 given by Devi $|11|$. We first necd to prove a 1 .cmma.

LEMMA.5. Let $\left(\mathrm{B}^{(\mathrm{i})}\right)$ be a sequence of infinite matrices such that (1.3) and (1.5), with a $\mathrm{nh}^{(\mathrm{i})}$ replaced by $b_{n k}(i)$, hold. Then, for every $z \in m_{0}$, we have $\mathbf{B} z=\mathcal{D} y$, where

$$
\mathbf{D}=\left(d_{n k}(i)\right)=\left(b_{n k}(i)-b_{n, k+1}(i)\right),
$$

and

If, further

$$
y=\left(y_{n}\right)=\left(\sum_{k=1}^{n} z_{k}\right) \in m
$$

$$
\lim _{n} \sum_{k} k_{n k}(i) l=0, \quad \text { uniformly in } i
$$

then $y \rightarrow O(D)$ and $z \rightarrow O(B)$.

PROOF: The first assertion follows from Abel's partial summation. The second one is a conseyucnce of the Result (3.2.1) given by Duran |10|.

We are now in a position to give the inequality mentioned above.

THEOREM.6. $\mathrm{L}^{*} \mathrm{~A} \leq \mathrm{W}^{*}$ if and only if $\mathrm{A}$ is F-regular and (2.8) holds.

Before proving the theorem we note that $\mathrm{W}^{*}$ is well-defined (sec Devi $\left.\mid 111\right)$.

We now come to the proof.

Suppose that $L^{*} A \leq W^{*}$. Since $W^{*} \leq L^{*}$, it follows from Theorem 4 that $\Lambda$ is $F_{\text {-regulal and }}$ that (2.8) holds.

Conversely suppose that $\Lambda$ is F-regular and (2.8) holds. By Theorem 4, we get

$$
u(x)=\inf _{z \in m_{0}} L^{*}(\Lambda(x+z)) \leq W^{*}(x)
$$

On the other hand

$$
L^{*}(\Lambda(x+z))=\limsup _{n} \sup _{i} \sum_{k} \frac{1}{n+T} \sum_{r=i}^{i+n} a_{r k}\left(x_{k}+z_{k}\right)
$$

Now write

$$
b_{n k}(i)=\frac{1}{n+1} \sum_{r=i}^{i+n} a_{r k}
$$


Since $\Lambda$ is F-regular, the reguirement of $L$.emma 5 is satisfied. Hence we have llat $7-3()(B)$ So. we get

$$
u(x) \geq \inf _{z \in m_{0}}\left\{L^{*}(\Lambda x)+L^{*}(\Lambda z)\right\}=I^{*}(\Lambda x)
$$

Hence the required conclusion follows from (2.10) and (2.11).

The following theorem is a generalization of Result VII given by Kuttner and Maddlox |12|.

THEOREM.7. Let $\|\mathcal{A}\|<\infty$ and $\|B\|<\infty$. Then $Q_{A}(x) \leq q_{B}(x)$ if only if $(B)$ is a Schur methol and

$$
\sum_{k} k_{n k}(i)-b_{n k}(i) l \rightarrow 0 \quad(n \rightarrow \infty, \text { uniformly in i) }
$$

PROOF. Since the proof uses the technique that Kuttner and Maddox used, |12|. we omit the details.

We conclude the paper with the following remark: Since no Schur method is regular. Theorem 7 includes the result that $Q_{A}(x) \leq g_{B}(x)$ is impossible when $(B)$ is a regular method. For eximple,

$$
\left.Q_{A}(x) \leq \mathfrak{L}(x) \quad \text { (for every } x \in m\right),
$$

is impossible.

\section{REFERENCES}

1. Lorentz, G.G. A contribution to the theory of divergent sequences. Acta Math. 80) (1948), 167-190).

2. King, J.P. Almost Summable sequences. Proc. Amer. Math. Soc. 17 (1966), 1219-1225.

3. Stieglitz, M. Eine verallgen meinerung des Begriffs Fastkonvergen7. Math. Janonn. 18 (197.3), 53-70.

4. Cooke, R.G. Infinitc Matrices and Sequence Spaces, Macmillian, 1950.

5. Maddox, I.J. Some analogues of Knopp's Core theorem. Internat. J.Math._and Math. Sci. 2 (1979), 605-614.

6. Simons, $S$. Ranach Limits, infinite matrices and sublinear functionals. J. Miath_ anal. Arpl 26 (1969), 640)-655.

7. Rhoades, B.E. Some properties of totally coregular matrices. Illinois J. Mallh. 1 (I)(1)). 518-525.

8. Schaefer, P. Core theorems for co-regular matrices. Illinois J. Math. 2 (196.5), 2(17-211.

9. Das, G. Sublinear Functionals and a Class of Conservative Matrices. Bull. Inst._Nath. Acitd. Sinica. 15 (1987), 89-106.

10. Duran, J.P. Infinite Matrices and Almost Convergence. Math. Z. 128 (1972), 75.83.

11. Devi, S.L. Banach Limits and Infinite Matrices. J. London Math. Soc. 12 (1976), 397-401.

12. Kuttner, B., Maddox, I.J. Inequalities between functionals on bounded sequencec. Indian J. Math. 25 (1983). 1-10. 


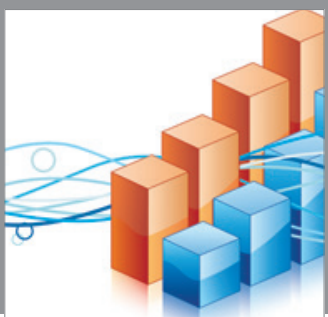

Advances in

Operations Research

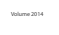

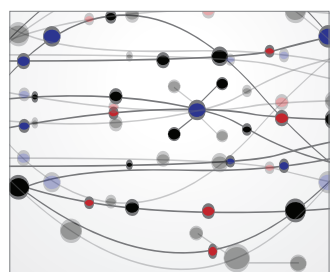

\section{The Scientific} World Journal
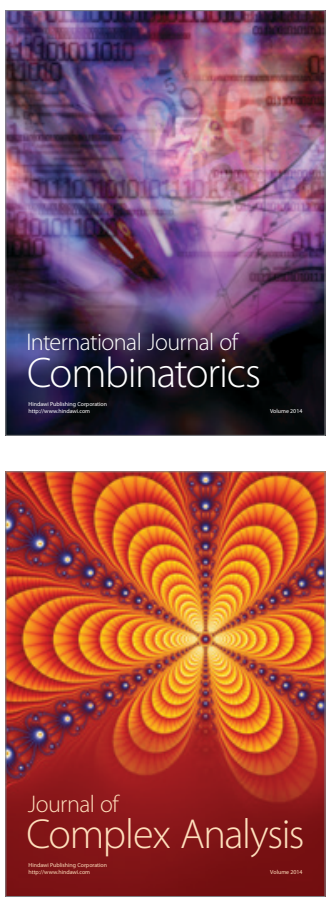

International Journal of

Mathematics and

Mathematical

Sciences
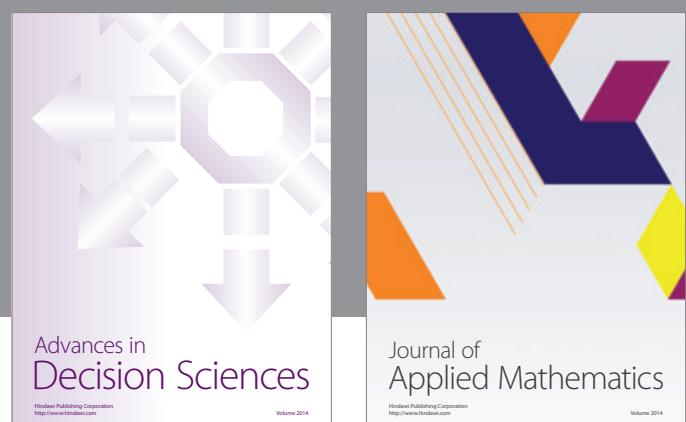

Journal of

Applied Mathematics
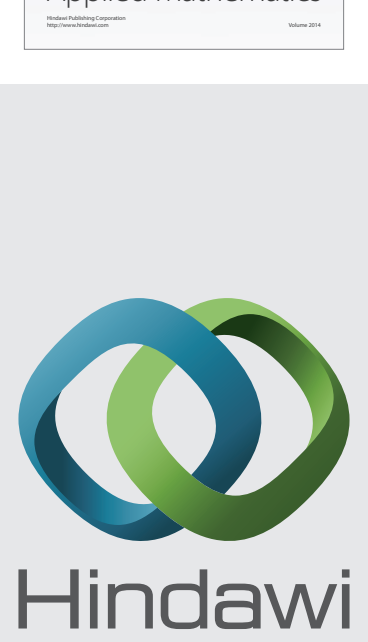

Submit your manuscripts at http://www.hindawi.com
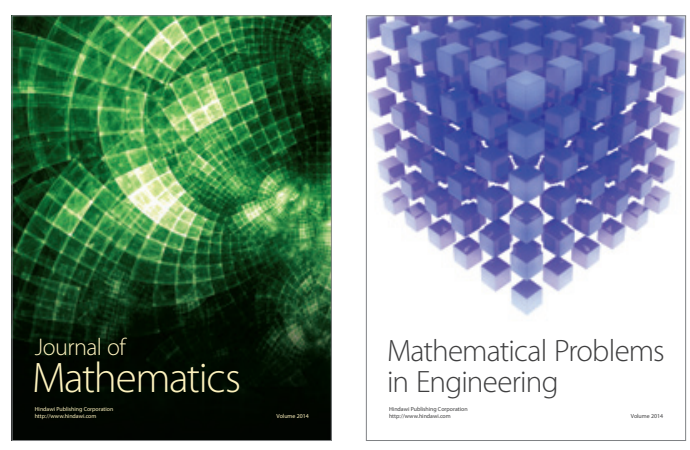

Mathematical Problems in Engineering
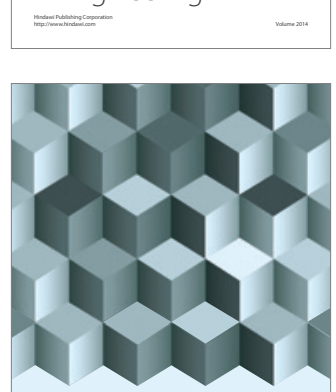

Journal of

Function Spaces
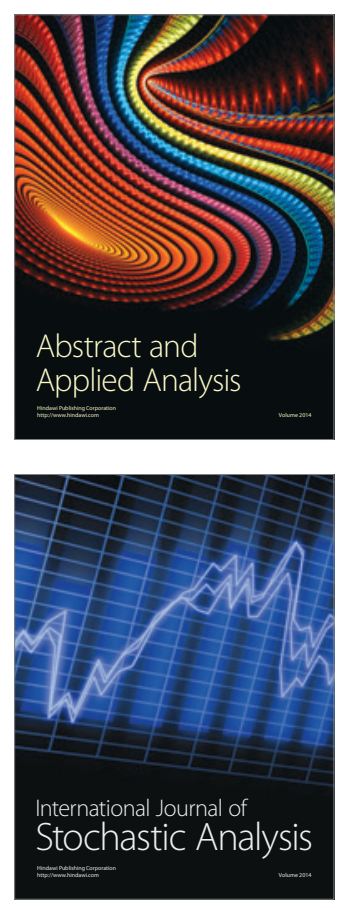

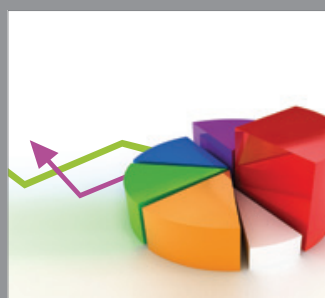

ournal of

Probability and Statistics

Promensencen
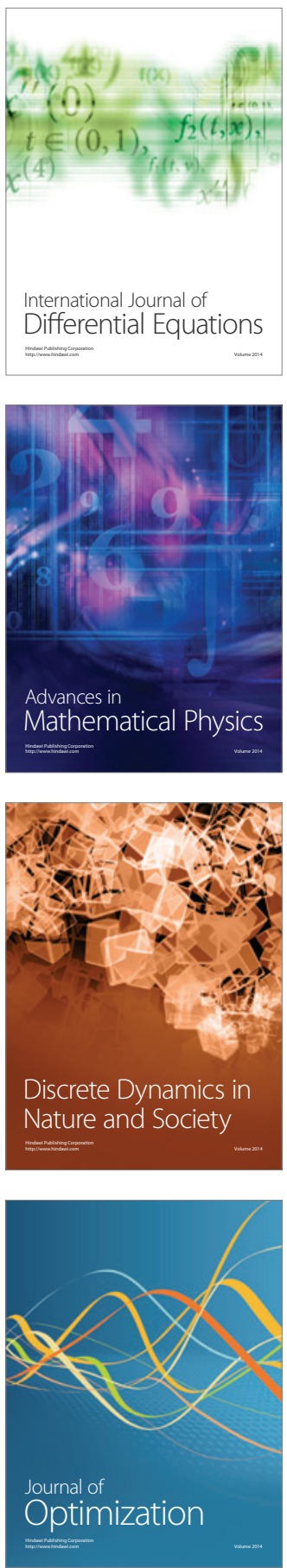